\title{
Introduction to Quantum Hall Effects
}

\author{
K.I. WYSOKIŃSKI* \\ Institute of Physics, M. Curie-Skłodowska University \\ Radziszewskiego 10, 20-031 Lublin, Poland
}

\begin{abstract}
The quantum Hall effect is a set of phenomena observed at low temperature in a two-dimensional electron gas subject to a strong perpendicular magnetic field. It manifests itself as a quantization of the nondiagonal elements $\left(\rho_{x y}\right)$ of the resistivity tensor accompanied by simultaneous vanishing $\rho_{x x}$ for ranges of the magnetic field. For the integer quantum Hall effect $\rho_{x y}=h / \nu e^{2}$, where $h$ is the Planck constant, $e$ - charge of an electron and $\nu$ is an integer, while for the fractional quantum Hall effect $\nu$ is a simple fraction. In spite of similar phenomenology deep and profound differences exist between these two effects. In the lecture the precision of the Hall quantization in the integer quantum Hall effect and briefly new types of quantum fluids observed in the fractional quantum Hall effect are discussed. Some recent theoretical and experimental discoveries connected with quantum Hall liquids are also mentioned.
\end{abstract}

PACS numbers: 73.43.-f, 73.40.-c, 73.50.Fq

\section{Introduction}

The phenomenon known as the Hall effect has been discovered in 1879 by Edwin Herbert Hall — at that time the graduate student at John Hopkins University in Maryland. One considers a metallic slab of thickness $d$ and applies magnetic field $\boldsymbol{B}$ perpendicularly to it in the presence of current $I$ flowing along the slab (see Fig. 1). As a result the voltage $V_{\mathrm{H}}$ appears across the sample. It is due to the Lorentz force $\boldsymbol{F}=q \boldsymbol{E}+q \boldsymbol{v} \times \boldsymbol{B}$ acting on a charge $q$ moving with velocity $v$ in a magnetic field $\boldsymbol{B}$. The Hall resistance $R_{x y}$ defined as a ratio of voltage $V_{\mathrm{H}}$ across the sample ( $y$-direction) to the current $I_{x}$ flowing along the sample (i.e. in $x$-direction) is found

$$
R_{x y}=\frac{B}{q N_{s}},
$$

where $N_{s}=n d$ is the number of carriers per unit area and $n$ - their concentration.

*e.mail: karol@tytan.umcs.lublin.pl 


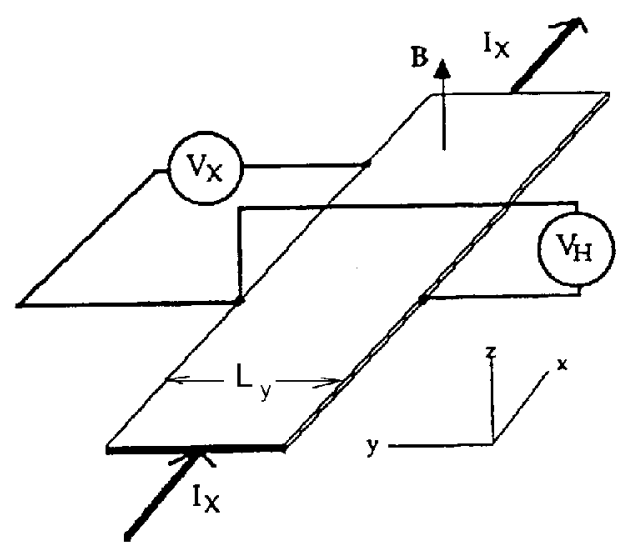

Fig. 1. The standard geometry used in the studies of the Hall effect in 3-dimensional samples and quantum Hall effects in 2-dimensional electron (or hole) gas.

Measurements of the Hall resistance (or more often the Hall coefficient $R_{\mathrm{H}}=$ $R_{x y} / B$ ) give information on the carrier density $n$ and their sign $q= \pm e$. For a given material (as characterized by $n, d$, and $q$ ) the measured value of $R_{x y}$ does depend on a magnetic field acting upon a sample. This simple fact is a basic principle of recently constructed quantum Hall probe microscopes [2] successfully used to measure magnetic field distribution around vortices in type II superconductors.

The quantum Hall effect (QHE) is a set of phenomena observed in a two-dimensional electron (or hole) gas. Similarly as in the Hall effect (which is purely classical phenomenon) one measures a voltage across the sample placed in a strong magnetic field. Again the voltage arises in response to the current flowing along the sample. The low temperature measurements [3] of the Hall resistance of a two-dimensional electron gas as a function of magnetic field have unexpectedly revealed strongly nonlinear behavior of $R_{x y}(B)$ for high enough fields. It manifests itself as a series of plateaux, extending over a range of magnetic fields in devices in which carrier concentration is kept constant (as in GaAs/AlGaAs heterostructures). To very high accuracy on a plateau one measures

$$
R_{x y}=\frac{h}{n_{\nu} e^{2}}=\frac{25812.807[\Omega]}{n_{\nu}}
$$

where $h$ is Planck constant, $e$ - electron charge and $n_{\nu}$ is a number.

We distinguish integer QHE (IQHE) when $n_{\nu}$ is an integer $1,2,3 \ldots$ and fractional QHE (FQHE) when the measured Hall resistance corresponds to $n_{\nu}=p / q$, and $p$ and $q$ are relatively prime integers. The appearance of the plateaux in $R_{x y}$ is in both cases accompanied by vanishing (in low enough temperatures) the longitudinal resistance $R_{x x}=I_{x} / V_{x}$, where $V_{x}$ is the voltage drop along the sample.

Even though the macroscopic manifestations of both effects is to a large extent the same, the physics behind them seem to be very different as will be seen in the following. 
In Sec. 2 a number of issues related to IQHE, inter alia its understanding and precision of resistivity quantization will be discussed. These include the importance of two-dimensionality, impurities, and the role of temperature and other aspects together with the breakdown of the effect. The fractional QHE is briefly introduced in Sec. 3. A short summary is given in Sec. 4.

\section{The integer quantum Hall effect}

The integer quantum Hall effect was discovered in 1980 by von Klitzing, Dorda, and Pepper [3]. Figure 2 shows a series of well-developed plateaus in the Hall resistance of the two-dimensional electron gas formed in the GaAsAlGaAs

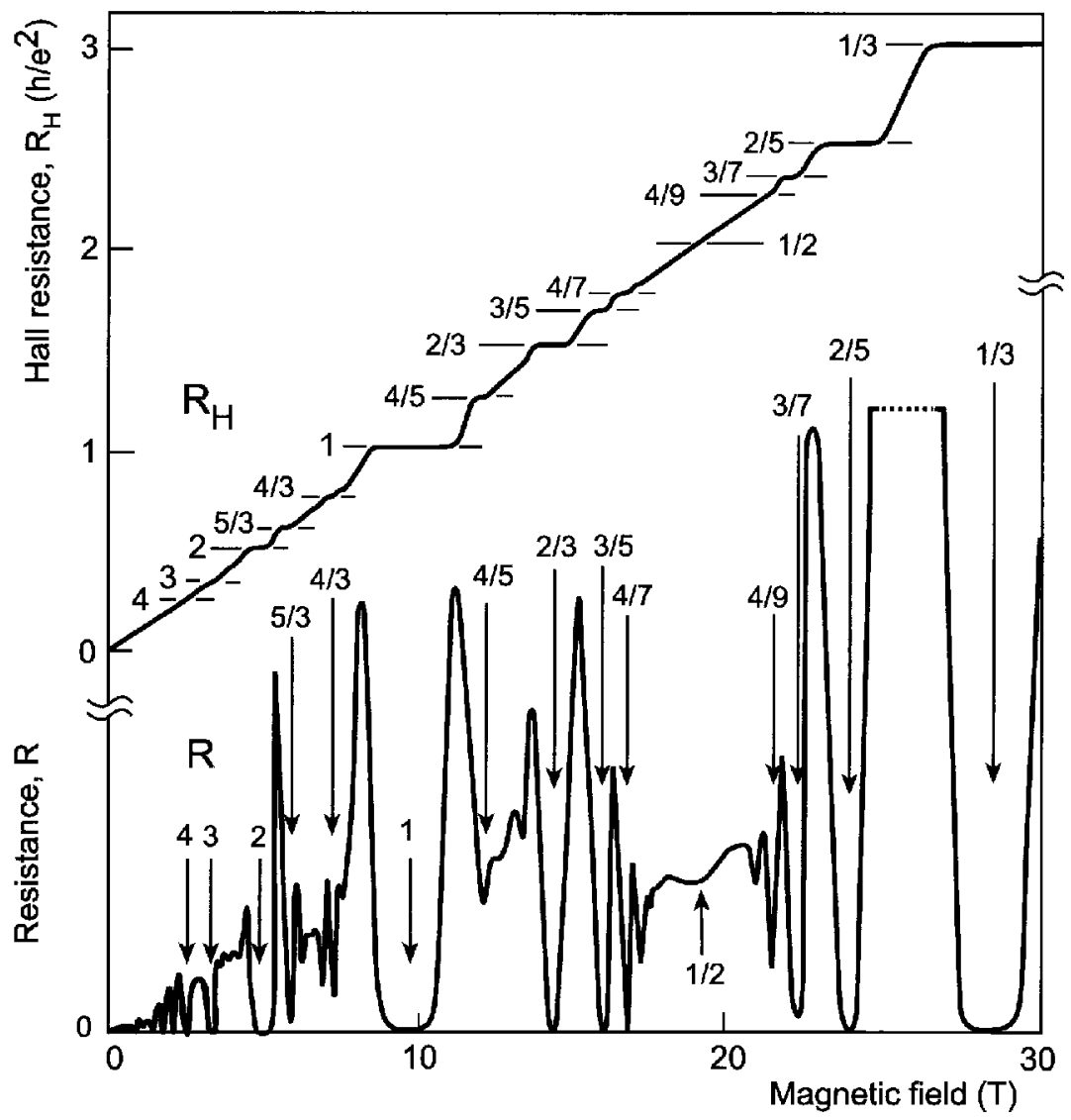

Fig. 2. The measurements on the very high mobility 2DEG showing integer and fractional quantum Hall steps reproduced from Ref. [19] with the author permission (upper part) and the oscillations of the longitudinal resistance (lower part). 
heterostructure. Remarkably the value of the Hall resistivity on the plateau is to high accuracy given by (2). In the original paper [3] the precision of $10^{-5}$ of quantization was observed. Shortly after the discovery of IQHE it turned out that the absolute precision of quantization of $R_{x y}$ is of the order of $10^{8}$ or better [4] and the agreement between measurements on different devices is of the order of $10^{10}[5]$. As a result the Hall resistance has been adopted as an international standard of resistance [6].

To understand the high precision of the measurement it is important to realize that in two dimensions the Hall resistance $R_{x y}$ and Hall resistivity $\rho_{x y}$ coincide (notation as in Fig. 1)

$$
R_{\mathrm{H}}=\frac{V_{\mathrm{H}}}{\mathrm{I}}=\frac{V_{\mathrm{H}} / L_{y}}{I / L_{y}}=\frac{E_{y}}{j_{x}}=\rho_{x y} .
$$

This means that a single and potentially very accurate "electric measurement" of $R_{x y}$ suffices for the determination of resistivity $\rho_{x y}$. To get the information on the other component of the tensor, i.e. the longitudinal resistivity $\rho_{x x}$, both the knowledge of $R_{x x}$ and sample dimensions $L_{x}, L_{y}$ are necessary as

$$
R_{x x}=\frac{V_{x}}{I_{x}}=\frac{E_{x}}{j_{x}} \frac{L_{x}}{L_{y}}=\rho_{x x} \frac{L_{x}}{L_{y}} .
$$

The measurements of $L_{x}$ and $L_{y}$, however, are never very precise. Note, in two dimensions the resistances and resistivities are expressed in the same units, namely ohms.

Note also that in two-dimensional system under consideration the symmetries $\sigma_{y y}=\sigma_{x x}, \sigma_{x y}=-\sigma_{y x}$ hold and the matrix elements of resistivity tensor $\rho_{\alpha \beta}$ are proportional to the corresponding elements of conductivity tensor $\sigma_{\alpha \beta}$, e.g. $\rho_{x x}=\sigma_{x x}\left(\sigma_{x x}^{2}+\sigma_{x y}^{2}\right)^{-1}$.

Three broad subjects connected with the IQHE will be discussed here. These include (i) the precision of the quantizations, i.e. the appearance of the flat plateaux for a range of magnetic fields, (ii) the transition region between the consecutive plateaux in which $\rho_{x x}$ takes on non-zero values and (iii) the breakdown of the IQHE.

\subsection{On a plateau: precision of the quantization}

As it is seen in Fig. 2 each plateau in $\rho_{x y}$ is accompanied by vanishing longitudinal resistivity $\rho_{x x}$. The absolute value of longitudinal resistance $R_{x x}$ measured in the Hall plateau is as low as $10^{-10} \Omega / \square$. This is a lower value than in any non-superconducting material. The IQHE has been observed in various systems containing two-dimensional gas of carriers. The results do not depend on a material, geometry of the sample, etc. The IQHE, however, is a low temperature effect. With increasing temperature the quantization accuracy is lowered, the plateaus become narrower and eventually vanish, the longitudinal resistivity takes on non-zero values. 
Before we start with theoretical explanation of the IQHE we have to note another aspect connected with the precision of quantization. Assuming a typical electron concentration in 2DEG of the order of $2 \times 10^{11} \mathrm{~cm}^{-2}$ and typical dimensions of the sample $260 \mu \mathrm{m} \times 400 \mu \mathrm{m}$ [7] we find the number of electrons in a two-dimensional channel $N \approx 2 \times 10^{8}$. Thus the precision of quantization is of the order of $1 / N$ instead of expected, on statistical grounds, much lower precision $1 / \sqrt{N} \approx 10^{-4}$ connected with fluctuations of physical parameters in the many body system.

\subsubsection{Two-dimensional electrons in crossed $E$ and $B$ fields}

It turns out that the IQHE can be understood solely in terms of single particle considerations. Thus we start with the ideal $2 \mathrm{DEG}$ in perpendicular magnetic $B$ and electric $E$ fields. Consider a typical Hall sample, as shown in Fig. 1 of area $L_{x} L_{y}$ placed in perpendicular magnetic field $\boldsymbol{B}=(0,0, B)$. The Hall voltage $V_{\mathrm{H}}$ across the sample is a source of the electric field $\boldsymbol{E}=(0, E, 0)$. The single electron Hamiltonian is given by

$$
\begin{aligned}
H= & \frac{1}{2 m^{*}}\left(-\mathrm{i} \hbar \frac{\partial}{\partial x}+e A_{x}\right)^{2}+\frac{1}{2 m^{*}}\left(-\mathrm{i} \hbar \frac{\partial}{\partial y}+e A_{y}\right)^{2} \\
& +e E y+V_{c}(x, y)+V_{\mathrm{imp}}(x, y)
\end{aligned}
$$

where $V_{\mathrm{c}}(x, y)$ is the confining potential, while $V_{\text {imp }}(x, y)$ represents the electron-impurity scattering potential. The Schrödinger equation $H \psi=E \psi$ with $V_{\mathrm{c}}(x, y)$ and $V_{\text {imp }}(x, y)$ can be solved only numerically. Without these two potentials the problem is exactly solvable. Using the Landau gauge for a vector potential $\boldsymbol{A}=$ $\left(-B y+\delta A_{x}, 0,0\right)$ (so $\boldsymbol{B}=\operatorname{rot} \boldsymbol{A}=(0,0, B)$ for $\delta A_{x}=$ const) and writing the electron wave function in the form of $\psi(x, y)=\left(1 / \sqrt{L_{x}}\right) \exp (\mathrm{i} k x) \varphi_{k}(x)$ we get equation

$$
\left[\frac{1}{2 m^{*}}\left(\hbar k-e B y+e \delta A_{x}\right)^{2}-\frac{\hbar^{2}}{2 m^{*}} \frac{\partial^{2}}{\partial y^{2}}+e E y\right] \varphi_{k}(x)=\varepsilon(k) \varphi_{k}(y),
$$

which is an equation for the displaced one-dimensional harmonic oscillator. Its solutions are expressed in terms of Hermite polynomials $H_{n}$ as

$$
\varphi_{n k}(y)=\frac{1}{\sqrt{2^{n} n ! \sqrt{\pi l}}} \exp \left[-\frac{\left(y-y_{k}\right)^{2}}{2 l^{2}}\right] H_{n}\left(\frac{y-y_{k}}{l}\right),
$$

where $n=1,2, \ldots, H_{n}(x)=(-)^{n} \exp \left(x^{2}\right)(\mathrm{d} / \mathrm{d} x)^{n} \exp \left(-x^{2}\right)$ is the $n$-th Hermite polynomial and $l=\sqrt{\hbar / e B}$ denotes magnetic length. The wave function $\varphi_{n k}(y)$ is seen to be centered around $y_{c}=y_{k}$, which in turn depends on the wave vector $k$. Explicitly

$$
y_{k}=l^{2} k-\frac{e E l^{2}}{\hbar \omega_{c}}+\frac{1}{B} \delta A_{x}
$$

and the eigenenergies become 


$$
\varepsilon_{n}(k)=\left(n+\frac{1}{2}\right) \hbar \omega_{c}+e E l^{2}\left(k+\frac{\delta A_{x}}{B l^{2}}\right)-\frac{1}{2} \frac{(e E l)^{2}}{\hbar \omega_{c}} .
$$

The spectrum has a linear dispersion. It consists of narrow bands separated by energy gaps $\hbar \omega_{c}=\hbar e B / m^{*}$, where $m^{*}$ is the effective mass of electrons in the system. For typical values of parameters $\hbar \omega_{c} \approx 1 \mathrm{meV}$. Note that for the vanishing electric field $(E=0)$ the spectrum consists of degenerate Landau levels. To find the degeneracy $g_{n}$ we assume the periodic boundary conditions in $x$ direction; $\psi(0, y)=\psi\left(L_{x}, y\right)$. This limits the allowed values of $k$ to $k=\left(2 \pi / L_{x}\right) r$, $r \cong 0, \pm 1, \pm 2, \ldots$. The distance $\Delta k$ between neighboring $k$ states is $\left(2 \pi / L_{x}\right)$. Because the center of wave function with the wave vector $k$ located at $y_{k}$ as given by (8) has to fall inside the sample width, i.e. $0 \leq y_{k} \leq L_{y}$, the total degeneracy $g_{n}$ of the $n$-th Landau level is found to be

$$
g_{n}=g=\frac{L_{y}}{l^{2} \Delta k}=\frac{L_{x} L_{y}}{2 \pi l^{2}}=\frac{L_{x} L_{y} e B}{h}=\frac{\phi}{\phi_{0}},
$$

where $\phi=L_{x} L_{y} B$ is the magnetic flux through the sample area and $\phi_{0}=h / e$ is the flux quantum. The ratio between the electron density $N / L_{x} L_{y}$ and the degeneracy $g$ is called the filling factor $\nu=N h / e B$.

\subsubsection{The appearance of a plateau: the role of disorder}

Imagine now that a number of Landau levels, say $n$, is fully occupied. It means that the electron density $N_{s}=g n / L_{x} L_{y}=n e B / h$. If this is plugged into Eq. (1) then we get

$$
R_{\mathrm{H}}=\frac{E}{e N_{s}}=\frac{B h}{e \cdot n e B}=\frac{h}{n e^{2}}
$$

in agreement with (2). Unfortunately, this does not yet explain the QHE, because we have only shown that for a very special density of electrons the Hall resistance takes on a very special value. Had one changed the electron density so the number of current currying states would increase and $R_{\mathrm{H}}$ would also change linearly with $B$ in complete disagreement with experiment.

In a real, impure system some states are localized and the number of current carrying states is smaller than the one found above. Moreover, all other states are scattered by impurities and there is no reason for the quantization. If however we observe quantum values of $\rho_{x y}$, as experiments show, it is so only because of some lucky compensation, which takes place. It turns out that the impurity potential which localizes some states, changes other states in such a way that they carry more current - exactly compensating for those that do not. This follows from the exact solution of the Hamiltonian (5) with the single short range impurity potential [8] and the scattering theory for general class of impurity potentials [9].

Somewhat different and in fact even more general explanation of the integer Hall plateaux and their independence of disorder has been proposed by Laughlin [10]. Let us imagine a two-dimensional system bent into a loop of circumference $L$ (see Fig. 3) with magnetic field piercing its surface and current $I$ flowing 


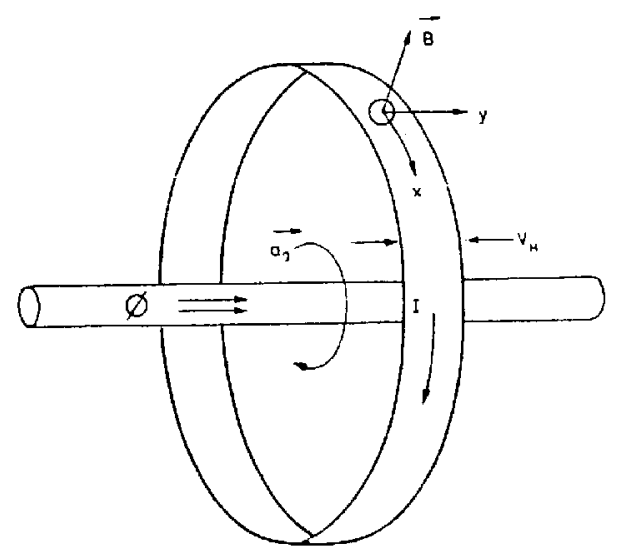

Fig. 3. The geometry used to explain the appearance of the quantum Hall plateaux.

around it. A long thin solenoid at the center of a loop is a source of flux $\phi$. By changing the flux $\phi$ one induces an electromotive force $V_{\text {ind }}=-\mathrm{d} \phi / \mathrm{d} t$, acting on the electrons at the cylinder surface. The work done, $V_{\text {ind }} / I \mathrm{~d} t$, equals the change in the internal energy $\mathrm{d} U$ of the system

$$
I=-\frac{\mathrm{d} U}{\mathrm{~d} \phi}
$$

and we find that the Hall current is given by the derivative of internal energy with respect to the flux through the solenoid. In the notation of the previous sections the change of the flux by $\delta \phi$ is equivalent to the change of the vector potential by $\delta \phi / L_{x}$. To see this note that $\delta \phi=\int \boldsymbol{B} \mathrm{d} \boldsymbol{S}=\int \operatorname{rot} \delta \boldsymbol{A} \mathrm{d} \boldsymbol{S}=\delta A L_{x}$. For localized states a change of the vector potential from $\boldsymbol{A}$ to $\boldsymbol{A}+\delta \boldsymbol{A}$ has no consequences, but for extended states of the type considered previously, this changes the wave function $\Psi_{n, k}(x, y)$ with a center of mass $y=y_{k}=k l^{2}-e E l^{2} / \hbar \omega_{c}+\left(\frac{1}{B}\right) \delta A_{x}$ (c.f. Eq. (8)) into the one centered at $y_{k^{\prime}}=k l^{2}-e E l^{2} / \hbar \omega_{c}+\delta A_{x}+\delta \phi / B L_{x}=y_{k+1}$ for $\delta \phi=h / e$ (a flux quantum). The net effect of the procedure is a transfer across the sample of one state (electron) per each occupied Landau level. The change of the internal energy is a product of the number of transferred electrons, their charge $(-e)$ and the voltage difference $V_{\mathbf{H}}$. The Hall current thus is

$$
I=\frac{\mathrm{d} U}{\mathrm{~d} \phi}=\frac{\Delta U}{h / e}=-n \frac{e^{2}}{h} V_{\mathrm{H}} .
$$

The quantization is exact whenever the Fermi level lies in a mobility edge. The above explanation works also for impure systems. To see this it is enough to consider the system in which edge regions are free from impurities and the interior is impure. By the above token one shows that a change in flux injects states into the disordered region on one side of the system and takes them away on the other. By charge conservation the end result is the transfer of electrons across the impure region. 
In fact it is the disorder in the system which breaks translational symmetry and leads to the quantization of Hall conductance. The change of the number of electrons in a system or changes of the magnetic field changes the position of the chemical potential but as long as it lies in the region of localized states the Hall current is constant and the free electron value of the resistivity remains intact $\rho_{x y}=h / n e^{2}$. This is consistent with experiment.

Moreover as long as the chemical potential lies in localized states the longitudinal resistivity is zero at zero temperature, because electrons from the current carrying states cannot be scattered across the gap, and there is no voltage drop in the direction of current flow. The longitudinal resistivity $\rho_{x x}$ thus vanishes. This explains the data.

The high precision of the quanntization in this approach is understood in the following way. The change of the flux in a system with a gap in the spectrum pumps a number of carriers across the sample. The Hall conductance resulting in this process essentially measures the number of carriers transferred.

\subsection{Between plateaux: localization-delocalization transition}

The standard scaling theory of localization predicts that in two dimensions all states are localized in thermodynamic limit no matter how weak the disorder is [11]. These results are evidently in conflict with the quantum Hall effect, which as we have seen requires the existence of extended states below the Fermi energy for its explanation. The energy which separates extended and localized states is called a mobility edge. In analogy with bulk systems the states in tails of Landau bands are localized. If the Fermi energy lies in a mobility gap the system is in the quantum Hall state with $\rho_{x x}=0$ and $\rho_{x y}$ quantized. If it is between lower $E_{c_{1}}$, and upper $E_{c_{2}}$ mobility edges then $\rho_{x x}$ takes on a non-zero value and $\rho_{x y}$ changes from a given quantized value to the next one (c.f Fig. 4).

Already from the early experiments it has been that with decreasing temperature the width of $\rho_{x x}$ peak $(\Delta B)$ decreased and slope of $\rho_{x y}$ increased pointing out that the region of extended states is very narrow if not of zero width. More detailed experiments have shown that $\Delta B \sim T^{\kappa}$ with $\kappa=0.42 \pm 0.04$ signaling that extended states do exist at the single energy value. The maximal slope of $\rho_{x y}$ as measured by $\mathrm{d} \rho_{x y} / \mathrm{d} B$ has been found to increase with the same exponent $\kappa$; $\mathrm{d} \rho_{x y} / \mathrm{d} B \sim T^{-\kappa_{v}}$ (Fig. 4). All this is consistent with the idea that lower and upper mobility edges coincide and extended states exist at a single energy. At all other energies the states are localized, i.e., their spatial extension $\xi$ is finite. The parameter $\xi$ is called a localization length. It depends on energy and for energies close to mobility edge is expected to diverge as $\xi \propto\left(E-E_{c}\right)^{-\nu}$. The measured value of $\nu \approx 2.34 \pm 0.04$ agrees quite well with theoretical estimations (see [12] for a review of various theoretical approaches and [13] for a classical calculation of the network model). 


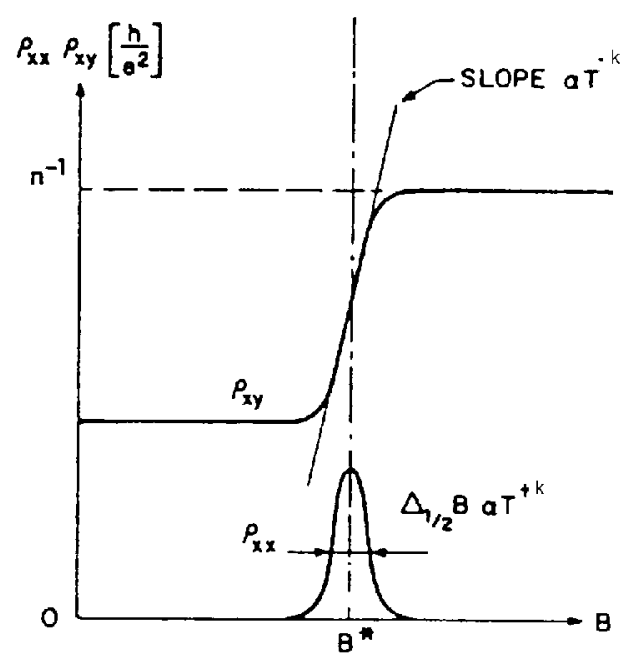

Fig. 4. Sketch of the transition region between two consecutive Landau levels. The slope of $\rho_{x y}$ scales with the same exponent as the widths of $\rho_{x x}$.

Each transition region between two consecutive IQHE steps in fact represents a quantum phase transition from localized to delocalized states. Quantum phase transitions take place at $T=0 \mathrm{~K}$. Experimentally they are studied at low but finite temperatures and manifest themselves as a narrowing down of regions, where $\rho_{x x} \neq 0$ and growing of the slopes $\left(\mathrm{d} \rho_{x y} / \mathrm{d} B\right)$. The scaling theory of phase transitions qualitatively explains the localization-delocalization transition in strong magnetic fields [14].

\subsection{Breakdown of $Q H E$}

Soon after the discovery of QHE it became evident that the increase in the current passing through the sample beyond a certain threshold value $I_{c}$ destroys the quantization [7]. Recently, the breakdown of QHE has become the subject of great interest and importance due to, already mentioned, application of quantum Hall devices to maintain national resistance standards. The subject will be discussed in detail later at this conference, so only a general introduction is given here.

In a typical QHE experiment one records the longitudinal voltage $V_{x x}$ as a function of magnetic field and finds, at each plateau, a region of dissipation-less flow (characterized by vanishing $V_{x x}$ ) over the range of fields $\Delta B=2 B_{c}$, where $B_{c}$ is called the critical field [15]. The breakdown of QHE manifests itself by the (often sudden) appearance of longitudinal voltage and the resistance $R_{x x}$ for $I>I_{c}$. The values of $I_{c}$ and $B_{c}$ depend on the filling factor (i.e. the plateau) and temperature $T$. Interestingly enough the observed temperature dependence of both 
$I_{c}$ and $B_{c}$ could be fitted by a slightly modified formula for the critical current in the Gorter-Casimir two-fluid model of superconductivity [15]. Sweeping back and forth the magnetic field in some samples reveals a hysteresis. The hysteresis, however, seems to disappear at elevated temperatures and after illumination [16]. The breakdown of QHE often shows up as a series of non-monotonic voltage steps recorded by changing $B$ at constant $T$ and for $I>I_{\mathrm{C}}$. These quantum steps have been proposed to result from the "turbulent" flow of carriers and are most probably due to their inter Landau level scattering [17].

\section{The fractional quantum Hall effect}

On phenomenological grounds FQHE looks like "ordinary" IQHE and is rationalized by Eq. (2) with $\nu$ being a fractional number. Originally the fractional steps with $\nu=1 / 3$ and $2 / 3$ were discovered in 1982 [18], during high field measurements aimed at the observation of Wigner crystallization of electrons.

The quality and the number of fractional steps depend on the mobility of carriers and temperature. In Fig. 2 a number of FQHE steps is visible [19]. The plateux are seen to appear not only in the lowest Landau level but also in a higher one. As it is easily seen most fractions have odd denominators. A more detailed analysis of these and other data shows that some sequences are particularly clearly developed. The sequences with $\nu=p /(2 p \pm 1)$ terminate at $\nu=1 / 2$. This is one of very special fractions with even denominators. At this filling factor one observes some features in $\rho_{x x}$ and none in $\rho_{x y}$.

Even though the FQHE appears to be the same as the IQHE, it shows the quantization of the Hall resistivity $\rho_{x y}$ accompanied by vanishing longitudinal resistivity $\rho_{x x}$ - its explanation is completely different. The first successful theory was published in 1983 by Laughlin [20]. He has proposed that the ground state of $N$ electrons in a partially filled Landau level is described by the many-body wave function

$$
\psi_{m}\left(z_{1}, z_{2}, \ldots, z_{N}\right)=\prod_{j<k}^{N}\left(z_{j}-z_{k}\right)^{m} \exp \left(-\frac{1}{4 l^{2}} \sum_{j}\left|z_{j}\right|^{2}\right)
$$

where $m=3$ and $z_{j}=z_{j}+\mathrm{i} y_{j}$ is the position of $j$-th particle in $(x, y)$ plane expressed as a complex number.

Some silent features of the Laughlin wave functions are as follows. Due to product terms the wave function keeps electrons apart and thus reduces their Coulomb energy making their motion highly correlated. There exists a gap between the ground state and excited states. The gap which now appears for partially filled Landau levels is due to interactions. The effect of disorder is presumably similar as in the IQHE. The disorder will localize the quasiparticles in the tails of bands allowing for a finite width of the plateaus. 
Till now there have been discovered more than 30 fractional Hall plateaus, and for most of them the appropriate wave functions can be deduced from Eq. (14). This wave function describes a new state of matter - an incompressible quantum liquid. The excitations over its ground state carry a fractional electric charge. These are extended objects the properties of which are not related to the electron properties but are determined by the interactions between electrons. In particular the energy required to create the quasiparticle has been estimated to be a fraction of the Coulomb interaction energy $e^{2} / l$ of two electrons being magnetic length apart from each other. The experimental search of fractional charge excitations started with the work of Clark and collaborators [21]. The most direct observations of these objects have been recently reported [22]. Note, however, that the fraction of the charge is different from the filling factor $\nu$ as shown in recent experiments [23] which measure the charge $\frac{1}{5} e$ on the Hall plateaus with $\nu=2 / 5$.

\section{Summary}

We have briefly discussed the issues connected with the Hall effects classic, quantum integer, and fractional - pointing out their universal aspects. The classic Hall effect is manifestly a universal phenomenon. The Hall resistivity $R_{\mathrm{H}}=B / q N_{s}$ depends only on the charge of carriers and their density per area. It does not depend on the disorder or the sample shape. Even if one punches holes in a sample, the measured parameters $q$ and $N_{s}$ are the same. This is not the case for longitudinal resistivity which depends on the impurities, their density, and other details.

This universal character of the Hall effect has shown its full glory in the precision of the von Klitzing effect (i.e. the IQHE). Due to a complete freeze out of the kinetic energy of electrons in the quantizing magnetic field the quantum character of transport shows up as a quantization of the Hall resistivity. The increase in temperature masks quantum effects and returns the classic behavior of resistivity, i.e. its proportionality to the magnetic field.

Each of the steps in FQHE signals formation of a new quantum liquid. Some of them are of particular interest as they do not show correlations of the type described by the Laughlin wave function.

The fractional charges have been directly observed in shot noise experiments. These objects possess also a fractional statistics which is related to topology of the system [24]. The field theory of fractional charge and statistics is a lovely subject intensively studied on various levels [25]. The topological aspect are very important in the understanding of the effect and the topology of the systems seems to play an interesting role as it has been shown in recent experiments in which a new collective state, sharing some similarities to ferromagnetism, Bose condensation, superfluidity and the Josephson effect and resulting from the phase coherence in bilayer system, has been discovered [26]. 
The readers interested in other aspects of these wonderful effects are advised to consult original literature or one of the books on the subject [27].

\section{References}

[1] N.W. Ashcroft, N.D. Mermin, Solid State Physics, Holt, Rinehart and Winston, New York 1976, Ch. 1.

[2] A.M. Chang, H.D. Hallen, L. Marriott, H.F. Heu, H.L. Kao, J. Kwo, R.E. Miller, R. Wolfe, J. van der Ziel, T.Y. Chang, Appl. Phys. Lett. 61, 1974 (1992).

[3] K. von Klitzing, G. Dorda, M. Pepper, Phys. Rev. Lett. 45, 494 (1980).

[4] L. Bliek, E. Braun, F. Melchert, P. Warnecke, W. Schlapp, G. Weimann, K. Ploog, G. Ebert, G. Dorda, in: Proc. 17th Int. Conf. on Low Temperature Physics, Eds. U. Eckern, A. Schmidt, W. Weber, H. Wühl, North-Holland, Amsterdam 1984, p. 411.

[5] A. Hartland, K. Jones, J.M. Williams, B.L. Galagher, T. Galloway, Phys. Rev. Lett. 66, 969 (1991).

[6] B.N. Taylor, Physics Today 42, 23 (1989).

[7] K. von Klizting, Rev. Mod. Phys. 58, 519 (1986).

[8] R.E. Prange, Phys. Rev. B 23, 4802 (1981).

[9] W. Brenig, Zeit. Phys. B 50, 305 (1983); J.T. Chalker, J. Phys. C, Solid State Phys. 16, 4297 (1983); D.J. Thoulless, J. Phys. C, Solid State Phys. 14, 3475 (1981); H. Aoki, T. Ando, Solid State Commun. 38, 1079 (1981).

[10] R.B. Laughlin, Phys. Rev. B 23, 5632 (1981); R.B. Laughlin, Rev. Mod. Phys. 71, 863 (1999).

[11] E. Abrahams, P.W. Anderson, D.C. Licciardello, T.V. Ramakrishnan, Phys. Rev. Lett. 42, 673 (1979).

[12] B. Huckestein, Rev. Mod. Phys. B 67, 357 (1995).

[13] K.I. Wysokiński, F. Evers, W. Brenig, Phys. Rev. B 54, 10720 (1996).

[14] A.M.M. Pruisken, in: Localization, Interaction and Transport Phenomena, Springer Series in Solid State Sciences, Eds. B. Kramer, G. Bergman, Y. Bruynseraede, Vol. 61, Springer Verlag, Berlin 1985, p. 127.

[15] L.B. Rigal, D.K. Maude, M. Potemski, J.C. Portal, L. Eaves, Z.R. Wasilewski, G. Hill, M.A. Pate, Phys. Rev. Lett. 82, 1249 (1999); L.B. Rigal, D.K. Maude, M. Potemski, J.C. Portal, L. Eaves, Z.R. Wasilewski, G. Hill, M.A. Pate, A.I. Toropov, Physica E 6, 124 (2000).

[16] T. Sanuki, K. Oto, S. Takaoka, K. Murase, K. Gamo, Solid State Commun. 117, 343 (2001).

[17] L. Eaves, Physica E 9, 45 (2001).

[18] D.C. Tsui, H.L. Stormer, A.C. Gossard, Phys. Rev. Lett. 48, 1559 (1982).

[19] H.L. Stormer, Rev. Mod. Phys. 71, 375 (1999).

[20] R.B. Laughlin, Phys. Rev. Lett. 50, 1395 (1983).

[21] R.G. Clark, J.R. Mallett, S.R. Haynes, J.J. Harris, C.T. Foxon, Phys. Rev. Lett. 60, 1747 (1988). 
[22] L. Saminedayar, D.C. Glattli, Y. Jin, B. Etienne, Phys. Rev. Lett. B 79, 2426 (1997); R. de Piccotto, M. Reznikov, M. Heibhem, V. Umansky, G. Bunin, D. Mahalu, Nature 389, 162 (1997).

[23] M. Reznikov, R. de Picciotto, T. Griffiths, M. Heizblum, V. Umansky, Nature 399, 238 (1999).

[24] D.J. Thouless, Topological Quantum Numbers in Nonrelativistic Physics, World Scientific, Singapore 1998, Ch. 7.

[25] F. Forte, Rev. Mod. Phys. 64, 193 (1992); E. Fradkin, Field Theories of Condensed Matter Physics, Addison-Wesley Publ. Co., Redwood City (CA) 1991, Ch. 10.

[26] J.P. Eisenstein, Physics World, June, 30, (2001).

[27] The Quantum Hall Effect, Eds. R.E. Prange, S.M. Girvin, Springer, Berlin 1986; M. Janssen, O. Viehweger, U. Fastenrath, J. Hajdu, Introduction to the Theory of the Integer Quantum Hall Effect, VCH, Weinheim 1994; T. Chakraborty, R. Petilainen, The Quantum Hall Effects, Springer Series in Solid State Sciences, Vol. 85, Springer, New York 1995. 